Journal of Case Reports 2020;10(2):132-135

\title{
Bilateral Tibial Spine Avulsion Fracture
}

\author{
Ittai Shichman, Dror Maor, Haggai Schermann, Guy Morag \\ Division of Orthopedic Surgery, Tel Aviv Sourasky Medical Center, Sackler Faculty of Medicine, Tel Aviv University, Tel Aviv, \\ Israel.
}

\section{Corresponding Author:}

Dr. Ittai Shichman

Email: ittaish@gmail.com

This is an Open Access article distributed under the terms of the Creative Commons Attribution License (creativecommons.org) licenses/by/3.0).

$\begin{array}{lll}\text { Received } & : & \text { March 14, } 2020 \\ \text { Accepted } & : & \text { June 17, } 2020 \\ \text { Published } & : & \text { June 30, } 2020\end{array}$

\begin{abstract}
Background: In skeletally mature patients, anterior cruciate ligament (ACL) avulsion fractures are considered uncommon. The objective of this paper is to present a rare case of a bilateral tibial spine avulsion in a skeletally mature one-month post-partum woman and to review literature on tibial spine avulsion fractures in the adult population. Case Report: A 30-year-old healthy female one-month post-partum suffered a crushing injury of both knees. She was diagnosed with Meyer-McKeever type II fracture on the right and type IIIA fracture on the left. According to literature review, female gender and post-partum ligamentous laxity were possible risk factors for this rare injury. Ten days after injury the fractures were treated surgically. Three months post-operatively she was pain free with full range of movements and had no clinical signs of knee instability. $\mathrm{X}$-rays demonstrated complete fracture union. Conclusion: The case presented concludes a rare diagnosis in a skeletally mature post-partum woman with high likelihood of pregnancy related osteoporosis and ligamentous laxity which probably were risk factors in the pathophysiology mechanism of injury.
\end{abstract}

Keywords: Anterior Cruciate Ligament, Arthroscopy, Intra-Articular Fractures, Knee Joint, Osteoporosis.

\section{Introduction}

Tibial spine avulsions are intra-articular fractures of the bony attachment of the anterior cruciate ligament (ACL) on the tibia that are most commonly seen in skeletally immature individuals from 8 to 14 years of age. It often occurs during athletic activity. Injuries that stress the ACL usually result in an avulsion of the ligament with the anterior tibial spine in children [1]. ACL avulsion fractures were traditionally considered uncommon in skeletally mature patients, and they are usually secondary to high velocity motor vehicle trauma accompanied by a high incidence of associated injuries [2]. Updated literature and better imaging modalities, however, suggest higher rates of occurrence among adults than those previously reported [3]. Reports on bilateral avulsions are very sparse, our literature search yielded only one documented case of a bilateral tibial spine avulsion in an adolescent [4]. We describe a rare case of a bilateral tibial spine avulsion in a skeletally mature female who was one-month postpartum and who sustained the injury in a low-speed motor vehicle accident.

\section{Case Report}

A 30-year-old physically fit and otherwise healthy female who was one-month post-partum suffered a crushing injury between a parked car and another car that was reversing. She was hit by the reversing car just below both knees, causing her to perform a sudden movement of flexion and rotation of her knees. She was brought to the nearest hospital emergency department (ED) where the physical examination revealed evidence of bilateral effusion. She was neurovascularly intact, and there was no 
clinical evidence of compartment syndrome. She had severe bilateral knee pain, which precluded the performance of a range of motion (ROM) and ligamentous examination. An X-ray raised the suspicion of bilateral spine avulsion fractures [Fig.1]. A computerized tomographic scan showed Meyer-McKeever type II tibial spine avulsion fractures in right knee and type IIIA for the left knee [Fig.2]. Her legs were immobilized with bilateral hinge braces locked in full extension to allow full weight bearing, and she was discharged home from the emergency department.

The patient was seen in our orthopedic outpatient clinic 10 days following the injury. She was using crutches to get around. Physical examination revealed a moderate amount of bilateral hemarthrosis, range of motion (ROM) of 10 to 120 on the left, and an ROM of 0 to 100 on the right. Active and passive ROM elicited pain. The results of ACL stability tests (Lachman and anterior drawer) were negative. The diagnosis was explained to the patient, and she was admitted for surgery after providing informed consent. The surgery consisted of bilateral knee arthroscopies. Direct arthroscopic visualization confirmed the integrity of the ACL fibers attached to a single avulsed fragment with no additional injury to the meniscus. The fragments were reduced arthroscopically and held with fiber-wire while drilling through an ACL aimer. The knees were evaluated arthroscopically, and the achievement of anatomic restoration was confirmed.

Post-operatively, the patient was allowed full weight bearing, with the right (dominant) leg immobilized in a hinged brace (between 0-45 degrees) and the left leg immobilized in full extension for the first two weeks in order to enable protected mobilization during early child care and breastfeeding. A physiotherapy regimen was initiated with bilateral continuous passive motion (CPM) between 0-60 degrees during the first two post-operative weeks. Hinged braces were then set at 0-60 degrees with bilateral CPM between

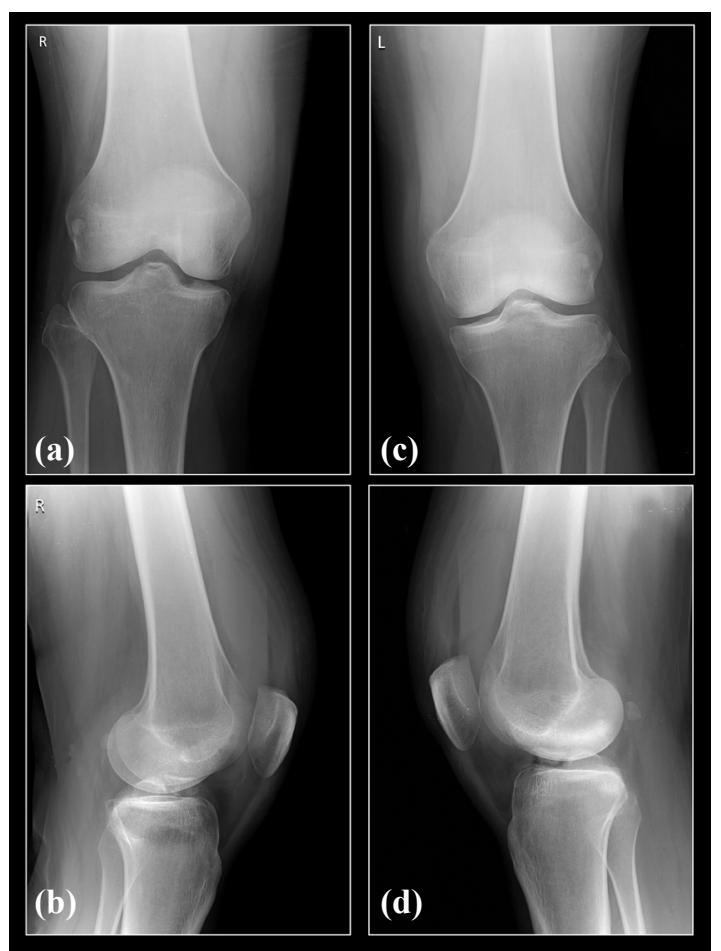

Fig.1: AP and lateral view of right $(a+b)$ and left $(c+d)$ knees on admission.

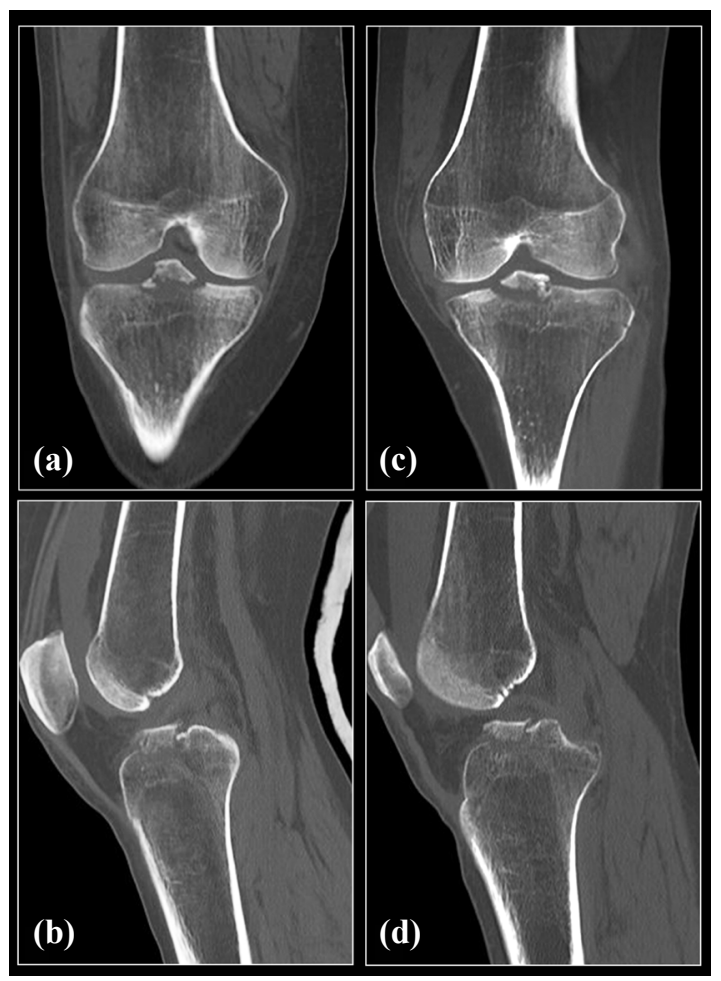

Fig.2: Coronal and sagittal CT scans of right $(a+b)$ and left $(c+d)$ knees on admission. 
0-90 degrees. The 6-week post-operative physical therapy included bilateral full ROM, bilateral active and passive reinforcement of the quadriceps muscles, stationary cycling, and transcutaneous electrical nerve stimulation. The 6-week postoperative $\mathrm{x}$-rays confirmed anatomic reduction of the avulsed fragments [Fig.3]. The 3-month postoperative x-ray confirmed united fragments. The patient was pain free, with full ROM, and had no clinical signs of knee instability.

\section{Discussion}

The pathophysiological mechanism of tibial spine avulsions in adults differs from that of the skeletally immature population. Overcoming the tensile bone strength at the ACL attachment requires high-energy trauma. The most common mechanism of injury is severe hyper-extension, which is usually associated with high-speed motor vehicle collisions, and adults with tibial eminence fractures have a higher prevalence of associated injuries [5]. These fractures are considered rare in the adult population. They were traditionally believed to represent the childhood equivalent of an ACL tear in adults [6]. It is now believed that they occur with much greater frequency in adults than previously reported, and, according to Kendall et al. [3], approximately $40 \%$ of all tibial spine fractures occur in adults. Bilateral tibial spine fractures, however, are extremely rare at any age. Our extensive literature search using Pubmed, Medline and the Cochrane Central Register of Controlled Trials (CENTRAL) yielded only one documented case of bilateral tibial spine fracture, and it was described in a skeletally immature individual [4].

There are multiple studies indicating a higher rate of ACL injuries in females compared to males $[6,7]$. The reason for this gender-related difference is multi-factorial. One is that higher concentrations of estrogen and relaxin-binding receptors are found in tendons and ligaments of women compared to men [8-10]. We consider

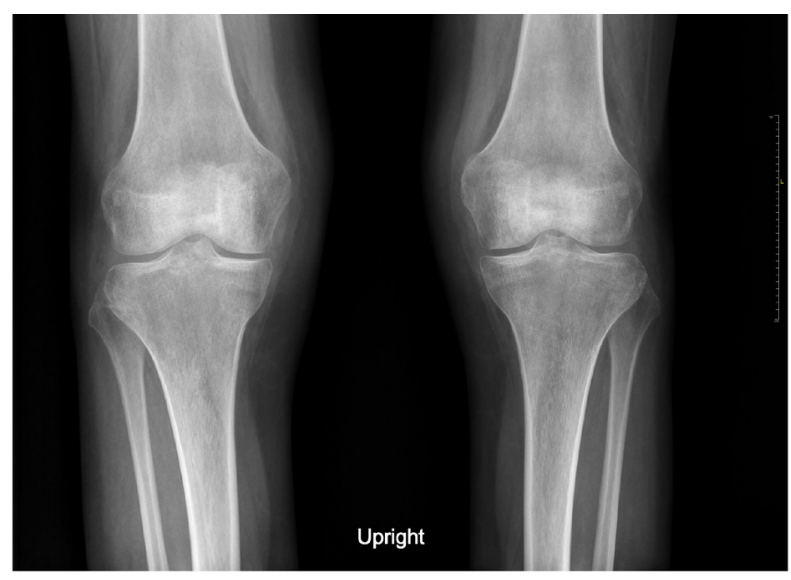

Fig.3: AP views 6 weeks following surgery.

that this would be further accentuated during pregnancy and the post-partum period. Moreover, the predominant musculoskeletal changes associated with pregnancy are related to weight gain and a subsequent change in the center of gravity (a forward shift), as well as a greater effect of circulating hormones on ligaments $[11,12]$. Increased levels of estrogen and relaxin during pregnancy leads to a greater increase in flexibility and decreased fibrillar cross-linking, resulting in greater joint laxity. A 20\% normal weight gain during pregnancy reportedly may increase the force on a joint by as much as $100 \%$ [12]. Altered joint biomechanics due to a lasting change in joint laxity in addition to normal weight gain could cause supraphysiologic stress on musculoskeletal tissues. Generalized ligamentous laxity in pregnancy has been correlated with shoulder dislocation, cruciate ligament tears, and patellar instability and dislocation [13]. The current information available on joint laxity during pregnancy and lactation indirectly suggests that these patients are at greater risk of injury and that they are more likely to have instability or subluxations in various joints [13]. We believe that this post-partum increase in laxity is a likely factor in our patient having sustained a bilateral tibial spine avulsion rather than rupturing her ACLs.

Arthroscopic reduction with suture fixation has become the preferred method of treatment for 
displaced or comminuted avulsion fractures of the tibial spine [5]. Direct intra-articular viewing now allows for anatomic reduction and treatment of any concomitant pathology. This surgical approach enables early mobilization of the knee, a shorter period of rehabilitation, and anatomic restoration of the articular surface.

\section{Conclusion}

The presented case description of a rare diagnosis in a skeletally mature postpartum woman suggests a high likelihood of pregnancy-related ligamentous laxity as a risk factor in the mechanism of bilateral tibial spine avulsion fracture associated with injury.

Contributors: IS and HS: manuscript writing; DM: manuscript editing; GM: imaging, diagnosis and manuscript editing. IS will act as a study guarantor. All authors approved the final version of this manuscript and are responsible for all aspects of the study.

Funding: None; Competing interests: None stated.

\section{References}

1. Meyers MH, McKeever FM. Fracture of the intercondylar eminence of the tibia. J Bone Joint Surg Am. 1970;52:1677-1684.

2. Patterson SP, Christiansen GB, Daffner RH. Avulsion fracture of the tibial eminence in an adult with a unique mechanism of injury. Radiol Case Reports. 2018;13:843847.

3. Kendall NS, Hsu SY, Chan KM. Fracture of the tibial spine in adults and children. A review of 31 cases. J Bone Joint Surg Br. 1992;74:848-852.

4. Andersen JW, Mejdahl S. Bilateral fracture of the tibial spine. Acta Orthop Belg. 1993;59:394-397.

5. Osti L, Buda M, Soldati F, Del Buono A, Osti R, Maffulli N. Arthroscopic treatment of tibial eminence fracture: a systematic review of different fixation methods. Br Med Bull. 2016;118:73-90.

6. Faryniarz DA, Bhargava M, Lajam C, Attia ET, Hannafin JA. Quantitation of estrogen receptors and relaxin binding in human anterior cruciate ligament fibroblasts. In Vitro Cell Dev Biol Anim. 2006;42:176-181.

7. Zelisko JA, Noble HB, Porter M. A comparison of men's and women's professional basketball injuries. Am J Sports Med. 1982;10:297-299.

8. Charlton WP, Coslett-Charlton LM, Ciccotti MG. Correlation of estradiol in pregnancy and anterior cruciate ligament laxity. Clin Orthop Relat Res. 2001;387:165170.

9. Chu SR, Boyer EH, Beynnon B, Segal NA. Pregnancy results in lasting changes in knee joint laxity. PM \& R. 2019; 11:117-124.

10. Wojtys EM, Huston LJ, Lindenfeld TN, Hewett TE, Greenfield MLVH. Association between the menstrual cycle and anterior cruciate ligament injuries in female athletes. Am J Sports Med. 1998;26:614-619.

11. DeMaio M, Magann EF. Exercise and pregnancy. J Am Acad Orthop Surg. 2009;17:504-514.

12. Ritchie JR. Orthopedic considerations during pregnancy. Clin Obstet Gynecol. 2003;46:456-66.

13. Miller EK, Tanaka MJ, LaPorte DM, Humbyrd CJ. Pregnancy-related ligamentous laxity mimicking dynamic scapholunate Instability: A Case Report. JBJS Case Connect. 2017;7:e54. 